\title{
Business Intelligence no agronegócio: um estudo de caso de implementação em uma startup
}

Business Intelligence in agribusiness: a case study of a startup

Ronan Guimarães Muynarsk ${ }^{*}$; Elisângela de Sousa Miranda²

\author{
${ }^{1}$ Agrocortex - Analista de Logística - Rua Dr Rafael de Barros, 210 - Paraiso - CEP 04003-040- São \\ Paulo (São Paulo), Brasil \\ 2 Universidade Federal da Grande Dourados FCBA - Professora Doutora - Rod. Dourados-Itahum, km 12 \\ CEP 79804-970 - Dourados (Mato Grosso do Sul), Brasil
}

\section{Resumo}

A base de dados das companhias, por muitas vezes, é originada de diversas fontes, dificultando o trabalho de transformação de dados em informações. A estruturação de um "Data Warehouse" [DW] e as ferramentas de "Business Intelligence" [BI] auxiliam essa transformação. O objetivo desse trabalho foi realizar um estudo de caso em uma startup do setor agroflorestal com o intuito de analisar e acompanhar a instalação das ferramentas de BI. Através de entrevistas com profissionais responsáveis, houve o acompanhamento da instalação dos sistemas operacionais e da estruturação do DW. O trabalho permitiu o acompanhamento da estruturação de cada uma das bases operacionais e a observação de melhorias na qualidade e na velocidade da informação disponível aos tomadores de decisão.

Palavras-chave: inteligência de mercado, setor florestal, tecnologia da informação

\begin{abstract}
The database of companies comes, most of the time, from different sources, making it difficult to turn data into information. On the other hand, structuring a data warehouse [DW] and business intelligence $[\mathrm{Bl}]$ tools can be a great support for the data work. Hence, a case study was carried out in an agroforestry sector startup, aiming to analyze and follow the installation of $\mathrm{BI}$ tools. The monitoring of the installation of the operating systems and structuring of the warehouse data was through interviews with responsible professionals. This work allowed the monitoring of the structure of each operational bases and the observation of improvements in the quality and the speed, giving available information to decision makers.
\end{abstract}

Keywords: market intelligence, forestry sector, information technology

\section{Introdução}

"Business Intelligence" $[\mathrm{BI}]$ pode ser definido como os processos de análise de informação dentro de uma empresa, com o intuito de melhorar seus processos de tomada de decisão e criação de vantagens competitivas (Jourdan, 2008).

Segundo Xu et. al (2007), o Bl é o processo de coleta de informação certa, no local certo, na hora certa, para entregar os resultados certos para as pessoas responsáveis pela tomada da decisão certa. Para Turban (2009), a expressão vai de encontro a um termo genérico onde se incluem arquiteturas, ferramentas, bancos de dados, aplicações e metodologias. Para o autor, a expressão é livre de conteúdo, podendo significar coisas diferentes de acordo com a conjuntura.

\footnotetext{
*Autor correspondente: <r.muynarsk@gmail.com> 
Turban (2009) acredita que os objetivos principais do BI são permitir acesso interativo aos dados (por muitas vezes em tempo real), proporcionar o manejo desses dados e fornecer aos analistas e gestores a capacidade de realizar análises que se enquadrem a realidade do negócio. $O$ autor ainda considerou que a análise de dados da situação e desempenho de uma empresa, tanto do seu histórico quanto da situação atual, permitem aos tomadores de decisão conseguirem alguns "insights" interessantes que podem embasar decisões melhores e mais aprofundadas. O Bl, então, baseia-se na transformação de dados em informações, depois em decisões e finalmente em ações.

O BI desempenha um importante papel, principalmente na área de negócios. Wong (2012) citou que os dispêndios nessa área, entre os diretores de tecnologia de informação, aumentaram ano a ano entre 2007 e 2012. Algumas pesquisas de mercado citadas pelo autor preveem crescimento nos investimentos nessa área até 0 ano de 2018.

Embora a área de $\mathrm{BI}$ desperte interesse entre as corporações, o sucesso em sua prática é bastante questionável. Ang e Teo (2000) relataram que cerca de 60 e $70 \%$ das companhias que experimentam adotar o BI falham por razões tecnológicas, culturais, organizacionais e de infraestrutura. Os autores relataram que algumas iniciativas de $\mathrm{BI}$ falham por não apresentarem acessibilidade aos usuários finais.

O BI se arquiteta em quatro grandes componentes: (1) o "Data Warehouse" [DW] que é um repositório que centraliza dados históricos, de modo que o acesso seja rápido e fácil e a sua manipulação para o suporte a decisões seja conveniente; (2) 0 ambiente de análise de negócios que permite aos usuários a criação de relatórios e consultas de acordo com suas necessidades de realizarem os dados; (3) o "Data Mining", que se trata de uma análise de dados, a qual procura padrões por muitas vezes ocultos em uma coleção de dados que podem ser usados para comportamentos futuros e que tem por base técnicas de estatística, matemática, inteligência artificial e aprendizagem automática para colher informações úteis ao negócio e; (4) O "Business Performance Management" se baseia na metodologia de "Balanced Scorecard" [BSC] que se "trata de uma estrutura para definir, implementar e gerenciar a estratégia de negócio de uma empresa” (Turban, 2009).

É importante salientar que o DW é composto por um conjunto de "Data Marts" o qual corresponde a um conjunto flexível de dados, ou seja, contém informações de acordo com o interesse ou assuntos específicos (Inmon, 1996). 
Para Wong (2012), o principal benefício do BI para uma empresa é a capacidade de oferecer informações necessárias exatas, incluindo uma visão em tempo real do desempenho do negócio, tanto geral quanto de uma pequena parte (a margem de lucro da empresa ou de um determinado item em específico). Turban (2009) relatou que essas informações são uma necessidade para qualquer tipo de tomada de decisão, para o planejamento estratégico e para a competitividade da empresa.

Esses mesmos autores concordaram que as principais vantagens da utilização do BI são a economia de tempo e de dinheiro, melhores estratégias e planos, melhores decisões táticas, geração de relatórios de forma mais rápida e precisa, processos mais eficientes e melhor serviço ao cliente.

As adoções de ferramentas de BI, no setor agroflorestal, possibilitam a melhoria da gestão do negócio. Dado histórico de fracassos em grandes projetos semelhantes ao da companhia, relatados por Silva et al. (2012), apontaram a necessidade de adotar melhores prática de gestão para a sobrevivência da empresa.

Diante do exposto, o presente estudo objetivou analisar e acompanhar, através de um estudo de caso, a implantação das ferramentas de Bl em uma startup de grande porte do setor florestal. Os objetivos específicos foram: (1) analisar a implantação dos "data marts" e seus devidos sistemas; (2) analisar a criação do DW e; (3) analisar as possibilidades de avanço na área de BI.

\section{Metodologia}

A metodologia empregada para o desenvolvimento dessa pesquisa foi estudo de caso. $O$ fato do objeto da pesquisa se embasar em condições contextuais, que podem ser pertinentes ao fenômeno analisado, respalda a escolha dessa metodologia. A característica de investigação empírica de um fenômeno real e com os limites entre os fenômenos analisados não definidos claramente entre si justifica essa escolha com base em Yin (2001).

O estudo foi realizado em um startup agroflorestal de grande porte de manejo florestal sustentável (ativo de 300 milhões de reais), com sua operação na cidade de Manoel Urbano, Acre, e sede administrativa na cidade de São Paulo, São Paulo. Fundada em março de 2014, a companhia implementou a gestão sustentável de floretas tropicais usando métodos de baixo impacto. O principal negócio da empresa é a extração e o desdobramento de madeira. 
A metodologia de coleta de informações e dados foi a pesquisa bibliográfica em artigos, livros, periódicos especializados, manuais dos sistemas, teses e dissertações e entrevistas com gestores das áreas envolvidas dentro da companhia. Os dados e informações coletados foram tratados e organizados para facilitar a análise. Em seguida foi elaborado o estudo de caso embasado em Yin (2001).

A organização e geração de dados se dividem em três sistemas operacionais: um sistema de gestão florestal desenvolvido sob demanda, um "Enterprise Resource Planning" [ERP] da SAP e um "Customer Relationship Management" [CRM] da "Insightly". No entanto, os três sistemas operacionais não eram interoperáveis.

A partir dessa caracterização surgiu a necessidade da criação de um unificador e organizador desses dados. A criação de um DW foi a solução encontrada para realizar uma única base, orientada por assunto e formato consistente.

As sequências de trabalhos no cronograma da companhia acompanhou o passo-a-passo da literatura: ferramentas de análise e visualização de dados, "data mining" e "business performance management".

As entrevistas com os profissionais responsáveis pelos três sistemas operacionais, assim como ao diretor responsável pela conclusão da instalação das ferramentas do $\mathrm{BI}$, foram conduzidas entre os meses de janeiro e maio de 2016. A preocupação central das entrevistas foi a busca da resposta de como cada elo do sistema funcionava.

\section{Resultados e Discussão}

\section{Sistema de Gestão Florestal}

Este sistema desenvolvido sob demanda teve primeira carga de dados de um completo inventário florestal. O inventário florestal é a base para o conhecimento da composição florística e determinação do volume remanescente e explorável da floresta a ser manejada (Francez et al., 2010).

O inventário foi esquematizado com pontos de Global Positioning System [GPS] para cada uma das árvores pertencentes a determinada Unidade de Produção Anual [UPA]. Desta forma, o trabalho consistiu em identificar espécies e volume de cada indivíduo, além de identificá-las com plaquetas de aço em números de série. Mapeada toda a UPA, a equipe de engenharia desenvolveu o Plano de Operação Anual $[P O A]$ que foi a segunda carga de dados.

Respeitadas as restrições das legislações ambientais em vigor (área de preservação permanente, espécies com manejo proibido, volume máximo explorado 
por unidade de área, entre outras) foram definidas quais árvores seriam abatidas, onde seriam construídas as estradas provisórias e onde seriam os pátios para toras.

A terceira carga de dados foi obtida pela operação florestal. Cada equipe de corte possuía uma subárea da UPA para explorar, e com o auxílio de um tablet equipado com GPS, a equipe identificou e abateu o indivíduo subindo a informação de corte pelo próprio equipamento.

O arraste das toras da floresta até os pátios foi realizado por equipamento pesado. O operador desta máquina possuía um equipamento similar ao das equipes de corte e também alimentava o sistema de operação florestal. No pátio foi realizado um romaneio, onde cada tora foi medida e as informações de volume do inventário foram confrontadas com as realizadas.

Nessa etapa foi adicionado a cada tora uma etiqueta com tecnologia RadioFrequency Identification [RFID] que acompanhariam a tora até o processo de serraria. No transporte de toras até a serraria cada caminhão também possuía esse mesmo equipamento de GPS adicionado a um leitor do RFID, alimentando mais um elo do sistema.

A quarta carga de dados do sistema foi feita pela operação industrial. O pedido migrado do CRM (que será descrito adiante) foi transformado em ordem de serviço. As toras foram serradas, os pedidos e o estoque remanescente separados e organizados para a secagem. Esse nível de detalhamento somadas as tecnologias de GPS e RFID utilizadas permitiu o cálculo de custos diretos para a operação de cada árvore, cada tora, cada elo da operação e cada pedido.

Esse nível de detalhe permitiu métricas apuradas de custos e margens, por espécie explorada, por pedido, até por indivíduo abatido além de desempenhos operacionais de cada equipe ou máquina. Os dados colaboram com ajustes e definições de metas para os próximos ciclos produtivos, identificando possíveis gargalos e folgas no processo produtivo.

A maior dificuldade encontrada na implantação desse sistema foi o treinamento da equipe desabituada com as plataformas desse gênero. A solução para contornar esse problema foi a modificação da remuneração comissionada das equipes vinculando-as com métricas de desempenho de qualidade das informações subidas ao sistema. Houve por parte dos desenvolvedores uma adaptação da plataforma para um formato mais visual, simples e intuitivo.

Outra adequação foi o sistema de carregamento de dados que em um primeiro momento eram carregados online via internet por rádio. Pelo sinal não alcançar todos 
os pontos da fazenda, essa metodologia se modificou para um carregamento no final do dia, assim que a equipe chegue na sede da fazenda.

\section{Enterprise Resource Planning [ERP]}

A solução adotada para a gestão financeira do negócio foi a compra do sistema "Business One" da SAP. Trata-se de um ERP projetado para negócios de médio porte. Essa ferramenta, além de auxiliar no atendimento de todas as responsabilidades fiscais e tributárias, possuiu uma facilidade de acesso aos dados e uma confiança nas consultas necessárias. A base de dados ficou armazenada na nuvem, que permitiu um acesso full time além de possibilitar que seus módulos rodassem juntos.

Esse sistema, no entanto, não se iniciou junto com a operação. Havia duas alternativas possíveis para o "Go Live": subir apenas os saldos dos fechamentos contábeis ou criar um histórico lançamento a lançamento. No que pesasse o custo de horas trabalhadas para a criação do histórico, além da prorrogação do "Go Live", a decisão foi de criar o histórico desde o início da operação. A vantagem de se ter toda a vida financeira da empresa documentada numa mesma base dados significou muito na estruturação de tecnologia de informação que se objetivou.

\section{Customer Relationship Management [CRM]}

O CRM procurou melhorar o relacionamento em todo o ciclo de vida de um cliente, identificando os clientes que mais contribuíram com o lucro e possibilitando a melhor gestão do intenso relacionamento e possibilitando a melhora dos resultados financeiros da companhia ${ }^{1}$.

A plataforma de CRM facilitou a gestão da carteira de clientes, das oportunidades de venda, dos pedidos e do desempenho de cada vendedor além de permitir uma customização de atendimento para cada cliente. Uma atenção especial nesse segmento se justificou por conta de o custo de conseguir um novo cliente ser maior que o custo de manter um cliente que já estava na carteira².

Cada cliente, dado suas características de compras, cotações e necessidades foi enquadrado e generalizado em um determinado perfil classificando-os em grupos. A definição desses grupos permite não ser necessário o tratamento específico para cada cliente, e sim para cada grupo.

\footnotetext{
${ }^{1}$ Martinelli, C.S. 2003. Implantação da estratégia CRM: estudo de caso em uma pequena empresa. 142 p. Dissertação de Mestrado em Engenharia Mecânica. Universidade Estadual de Campinas. Campinas, São Paulo, Brasil.

${ }^{2}$ Martinelli, op. cit.
} 
No caso abordado, a geração de dados nesse sistema foi de responsabilidade do setor comercial e de logística da companhia. O acompanhamento de cada etapa do pedido (serragem, secagem, expedição, transporte terrestre, despacho aduaneiro e transporte marítimo) foi realizado dentro desse sistema. Dessa forma, através de métricas de desempenho, os pedidos foram fiscalizados de perto por todos os gestores envolvidos.

Por outro lado, o acompanhamento das cotações lançadas no mercado facilitou o trabalho dos vendedores na análise individual para novas cotações. Informações como quais e quantas cotações foram vencidas ou perdidas, quanto tempo em média demora para se ter resposta e quais preços e produtos foram ofertados, possibilitam a mitigação de erros de cotações múltiplas, facilitando as tomadas de decisão cotidianas dos vendedores.

\section{Data Warehouse [DW]}

Ao término da arquitetura de sistemas operacionais, o nível estratégico da companhia notou a dificuldade de analisar dados de fontes distintas. Apesar de cada base trazer em seus "data marts" informações necessárias para medir seu desempenho, a visão global do negócio ficava prejudicada com essa estrutura plural.

A solução proposta pela área de tecnologia da informação foi o investimento em um armazém central de dados mais relevantes de múltiplas fontes, onde pudesse orientar pesquisas por assunto, variasse ao longo do tempo e permitisse consultas rápidas e de grande volume de informação: um DW.

O projeto ganhou força com o fim da fase pré-operacional da companhia, onde as bases de dados do CRM e do sistema de gestão florestal começaram a se tornar mais robustas, além de crescer a demanda por informações que, por muitas vezes se provinham de mais que uma base.

A solução passava por duas aquisições: amplo espaço de armazenamento na nuvem e um computador de alta capacidade de processamento de dados. Tomada a decisão de avançar na área, o próximo desafio foi a parametrização das diferentes bases de dados no DW. Essa questão foi solucionada por consultores de programação de banco de dados.

A implantação do DW permitiu a análise cúbica de dados que eram realizadas com planilhas eletrônicas para a obtenção de informações de diversas fontes. Um exemplo de informações que necessitavam de dados de mais de uma fonte foi o cálculo da margem líquida por espécie. 
Para obter esse importante indicador, se fez necessário extrair dados do sistema de gestão florestal, do ERP e do CRM. Esses dados eram concentrados em uma planilha eletrônica e cruzados para a extração da informação necessária.

Até o término dessa pesquisa em maio de 2016, a empresa estruturava o ambiente de análise de dados do DW. Como tratado na sessão introdutória deste trabalho, o "Business Intelligence" acarreta em adquirir dados e informações de diversas fontes, organizar em uma DW e utilizá-los como ferramentas nas tomadas de decisão.

Essa aplicação analítica poderia ser considerada um avanço na direção à sofisticação em relação ao oferecimento de simples ferramentas ou técnicas de análise de dados. Elas permitiriam a automatização do pensamento e de parte da tomada de decisão, além de disponibilizar o uso comum de técnicas quantitativas com maior complexidade tais como análise de regressão multivariada, "data mining", programação não linear ou inteligência artificial.

O resultado das solicitações viriam em forma de relatórios, previsões, alertas ou apresentações gráficas, da forma que for mais útil para a tomada de decisão. Algumas aplicações mais sofisticadas de BA que seriam instaladas incluem atividades como orçamentos, alocação de recursos, modelagem financeira, e inteligência competitiva. Essas ferramentas mais elaboradas envolveriam fatores como modelagens de decisão, análise de desempenho de negócio, métricas, ferramentas de reengenharia, entre outros.

A conclusão da instalação do DW também abriu caminho para esforços de "data mining". Essa aplicação do BI resumiu na utilização do banco de dados para extrair informações não triviais, que em um ambiente de simples análise ou consulta não são identificadas. A utilização do "data mining" dessa aplicação pode colocar a empresa em vantagens comparativas ao mercado. O cronograma da companhia data em janeiro de 2017 o início das atividades de "data mining".

Outra aplicação do BI foi o Business Process Management [BPM]. O foco dessa aplicação foi transformar as estratégias e objetivos em planos, de modo a monitorar o desempenho do seu andamento.

O BPM possui um conjunto integrado de processos, metodologias, aplicações e métricas criadas para mensurar e monitorar o desempenho financeiro e operacional de uma companhia. O BPM pode ser visto como o resultado do BI, incorporando muitas de suas tecnologias, técnicas e aplicações. 
Apesar de seu princípio não ser novo (dividir estratégia em planejamento), o BPM adiciona a interação desses processos, metodologias, sistemas e métricas, buscando que as empresas foquem no processo como um todo em vez de um projeto por vez (Han et al., 2012).

\section{Conclusão}

O trabalho permite a análise e o acompanhamento através do estudo de caso da implementação dos sistemas operacionais, seus devidos "data marts" e a construção do "Data Warehouse" [DW] além da construção da base parra a instalação das ferramentas e aplicações do "Business Intelligence" [BI].

Essa estruturação de tecnologia da informação possibilita a análise de desempenho da empresa mais apurada e menos pulverizada, o início dos esforços em "data mining" e a maior confiabilidade nas informações com mais de uma fonte.

As principais vantagens da utilização do $\mathrm{BI}$, indicadas pela literatura, são alcançadas parcialmente pela empresa. Apesar do estudo não ter acompanhado até a conclusão da instalação de suas ferramentas, alguns progressos são notados de imediato.

O maior avanço da Companhia é a centralização dos dados no DW, reduzindo tempo para geração de relatórios e facilitando análise cúbica. As oportunidades abertas com o BI para esforços com "data mining" e a instalação do "Business Process Management" [BPM], apesar de não observadas, se tornam possíveis a partir dessa implantação.

Devido ao curto espaço de tempo, não são notadas modificações nas tomadas de decisão no nível estratégico.

\section{Referências}

Ang, J.; Teo, T.S.H. 2000. Management Issues in Data Warehousing: Insights from the Housing and Development Board. Decision Support Systems 29: 11-20.

Francez, L.M.B; Souza, D.V.; Takahana, C.L.I.; Barros, P.L.C. 2010. Manual para análise de inventário florestal e equação de volume em projetos de manejo florestal sustentável. Disponível em:

<http://www.sema.pa.gov.br/anexos_legislacao/Anexoll.pdf>. Acesso em: $27 \mathrm{fev}$. 2015.

Han, J.; Kamber, M.; Pei, J. 2012. Data Mining: Concepts and Techiniques. Elsevier, Waltha, Massachusetts, USA. 
Inmon, W.H. 1996. The data warehouse and data mining. Communications of the ACM 39(11): 49-50.

Jourdan, Z.; Rainer, R.K.; Marshall, T.E. 2008. Business intelligence: An analysis of the literature. Information Systems Management 25: 121-131.

Silva, L.F.; Silva, M.L.; Cordeiro, S.A. 2012. Análise do mercado mundial de madeiras tropicais. Política Agrícola 21: 1-7.

Turban, E.; Sharda, R.; Aronson, J. E.; King, D. 2008. Business Intelligence: A Managerial Approach. Prentice Hall, Upper Saddle, New Jersey, USA.

Wong, K. L; Chuah, M. H. 2012. Construct na Entreprise Business Intelligence Maturity Model (EBI2M) Using na Integrantion Approach: A Conceptual Framework. p. $1-14$. In: Mircea, M. Business Intelligence - Solution for Business Development. Intech. Disponível em < http://www.intechopen.com/books/business-intelligence-solution-forbusiness-development>. doi: 10.5772/2352.

Xu, L.; Zeng, L.; Shi, Z.; He, Q.; Wang, M. 2007. Research on Business Intelligence enterprise computing environment. Paper presented at the IEEE International Conference on Systems, Man and Cybernetics, Montreal, Quebec, Canada.

Yin, R.K. 2001. Estudo de Caso: Planejamento e Métodos. Bookman, Porto Alegre, Rio Grande do Sul, Brasil. 